konsolidieren - Neuverschuldung auf Null reduzieren` (Drucksache i6/277I, Absatz 4), IMK Policy Brief, September 2009.

Truger, A., Will, H., Köhrsen, J. (2009a): Die Schuldenbremse: Eine schwere Bürde für die Finanzpolitik. Stellungnahme des IMK in der Hans-Böckler-Stiftung im Rahmen der öffentlichen Anhörung des nordrhein-westfälischen Landtags am I7. September 2009, IMK Policy Brief, September 2009.

Truger, A., Eicker-Wolf, K., Will, H., Köhrsen, J. (2009b): Auswirkungen der Schuldenbremse auf die hessischen Landesfinanzen. Ergebnisse von Simulationsrechnungen für den Übergangszeitraum von 20Io bis 2020, IMK Studies, Nr. 6, Düsseldorf.

Vesper, D. (2007): Staatsverschuldung und öffentliche Investitionen, IMK Policy Brief, November 2007.

\title{
Die Kontroverse Frankfurter Allgemeine Zeitung contra Handelsblatt. Einige Bemerkungen zu einem methodologischen Schlagabtausch anno 2009
}

The controversy between Frankfurter Allgemeine Zeitung and Handelsblatt. Some remarks on a methodological exchange in the year 2009

\section{Kurt Rothschild}

Auseinandersetzungen über methodologische Fragen gibt es in allen Wissenschaften. Fragen bezüglich der Zweckmäßigkeit, der Berechtigung, der 'Wissenschaftlichkeit ‘ alternativer Methoden werden fallweise aufgegriffen und mit viel Animo diskutiert. Hitzige Auseinandersetzungen zwischen Freudianer|inne|n, Adlerianer|inne|n und anderen Psycholog|inn|en, zwischen Schulmedizin und Homöopathie, zwischen theoretischen und Experimentalphysiker|inne|n bieten signifikante Beispiele für solche Konfrontationen. Aber in manchen Wissenschaftszweigen spielen solche Auseinandersetzungen fallweise eine größere Rolle als in anderen. Zu diesen zählt sicher die Wirtschaftswissenschaft. Sie ist die einzige, in der das Wort `Methodenstreit ‘ eine eigenständige Bedeutung mit Bezug auf eine frühe fundamentale Auseinandersetzung erhalten hat, die Auseinandersetzung zwischen Menger und Schmoller bzw. zwischen österreichischer und historischer Schule. In diesem ursprünglichen Methodenstreit ging es vor allem um die Frage, wie weit ökonomisches

* Österreichisches Institut für Wirtschaftsforschung, Wien.

(C) INTERVENTION 7 (I), 20I0, 24-3I 
Geschehen auf Grund allgemein gültiger Gesetzmäßigkeiten im menschlichen Verhalten und darauf aufbauender Axiome verstanden werden kann (österreichische Schule), oder ob ein sich ständig verändernder öknomischer Bereich nur im Rahmen einer dynamischen und gesamtgesellschaftlichen Analyse (historische Schule) erklärbar ist. Methodologisch bedeutete die österreichische (bzw. die verwandte neoklassische) Schule eine Orientierung an den Naturwissenschaften mit ihren deduktiv abgeleiteten Gesetzmäßigkeiten, während die historische Schule ihr Vorbild in den induktiv-deskriptiven, ,verstehenden Methoden der Geschichtsschreibung fand.

Bekannterweise ging diese österreichisch-deutsche Auseinandersetzung weltweit mit einem Sieg Mengers aus, ohne allerdings die damals aufgeworfene Problematik verschiedener Zugänge zur Erklärung und Prognose ökonomischer Prozesse zu überwinden. ${ }^{I}$ In vielen späteren methodologischen Auseinandersetzungen (Keynes//Klassik', orthodoxe/heterodoxe Ökonom|inn|en etc.) finden sich Echos zu dieser Dichotomie. Dies gilt auch für den methodologischen Schlagabtausch des Jahres 2009, von dem hier die Rede ist.

Dieser unerwartet aufgetretene Schlagabtausch ist aus zwei Gründen bemerkenswert: Erstens, weil im Gegensatz zu den in diversen Aufsätzen unregelmäßig dahinplätschernden methodologischen Bemerkungen einzelner Ökonom|inn|en hier zwei große Gruppen von Ökonom|inn|en (aus dem deutschsprachigen Bereich) programmatisch Stellung beziehen; und zweitens, weil ihre Stellungnahmen in zwei führenden Tageszeitungen - der Frankfurter Allgemeinen Zeitung (FAZ) und dem Handelsblatt (HB) - der breiten Öffentlichkeit vorgelegt wurden. ${ }^{2}$

Mit dieser Konfrontation beschäftigen sich die folgenden Bemerkungen. Zunächst werden die inhaltlichen Gegensätze der beiden Gruppen kurz einander gegenübergestellt und kommentiert, was übrigens auch bereits Stoff breiterer Beiträge in Zeitungen, Zeitschriften und im Internet ist. Daran schließt sich eine Überlegung zum Stellenwert der beiden Positionen in der generellen methodologischen Debatte an.

I Alfred Marshall, einer der Väter der Neoklassik und insofern `Mengerianer`, war sich der Bedeutung der `Historiker` wohl bewusst. In seinen Principles schreibt er mit Bezug auf die deutschen Ökonom|inn|en der historischen Schule: »They have given their chief attention to the historical treatment of the science, and its application of the condition of German social and political life. [...] They have made careful and profound analyses which add much to our knowledge, and they have greatly extended the boundaries of economic theory." (Marshall 1920/1994: 634)

2 Einen bedeutsamen öffentlichen methodologischen Auftritt gab es allerdings bereits früher. Im Jahre 2000 verfassten Pariser Ökonomie-Student|inn|en eine Petition, dass im Ökonomiestudium nicht nur die Neoklassik zu Wort kommen solle, sondern auch andere Theorien und Methoden sowie die Geschichte der Nationalökonomie. Diese Petition wurde in zahlreichen führenden Zeitungen und Zeitschriften veröffentlicht und fand ein so breites Echo, dass das Unterrichtsministerium eine Kommission zur Untersuchung des Problems einsetzte. Der französische Appell fand anschließend Unterstützung an Universitäten in England und in den USA und führte zur Gründung der >Post-Autistic Movement «, die im Internet ein kostenfreies Journal veröffentlicht, den `Real World Economic Review` (ursprünglich >Post-Autistic Economic Review`; siehe http://www.paecon.net sowie die Vorstellung des Arbeitskreises Post-Autistische Ökonomie in Intervention Nr. 2/2005). 
Ausgelöst wurde die hier behandelte Auseinandersetzung durch ein konkretes Ereignis, nämlich Beschlüsse der Universitäten Köln und Freiburg, einige ökonomische Lehrstühle, die bisher unter dem Titel ,Wirtschaftspolitik Zugang zu ökonomischen Problemen vertraten, in Abteilungen umzuwidmen, die sich mit Titeln wie `Makroökonomie` und dergleichen enger an der vorherrschenden `reinen klassischen Theorie und deren Methoden orientieren sollten. Kritik an diesem Vorhaben von einigen Ökonom|inn|en führte dann zu dem Schritt in die Öffentlichkeit durch eine von 83 Ökonom|inn|en unterzeichnete Stellungnahme in der FAZ vom 5 . Mai 2009, der am 8. Juni eine geharnischte Gegenposition, unterzeichnet von I88 Ökonom|inn|en im HB, folgte. Der Inhalt dieser beiden `Pamphlete` soll nun stark komprimiert zusammengefasst werden (wobei der teilweise gereizte Ton der Beiträge verloren geht).

In ihrem relativ kurzen FAZ-Beitrag betonen die 83 Unterzeichner|innen die speziellen Aufgaben, welche die Wirtschaftswissenschaft zu erfüllen hat, und die wichtige Rolle, welche die bedrohten wirtschaftspolitischen Lehrstühle in diesem Zusammenhang spielen. Als wichtig werden praktische wirtschaftspolitische Fragestellungen (Staatsanteil, Finanzen, Verteilung, Stabilität etc.), eine Berücksichtigung und kritische Analyse wirtschaftspolitischer Institutionen und wirtschaftspolitischer Instrumente und die Hinterfragung der Effizienz wirtschaftspolitischer Maßnahmen genannt. Hinzugefügt wird dann, dass »die systematische Analyse solcher wirtschaftspolitischen Fragestellungen ohne normative Grundlagen nicht möglich ist«. Der vorherrschenden Wirtschaftswissenschaft wird vorgeworfen, dass ihre Methodologie vor allem darauf ausgerichtet ist, "aus jeweils gewählten Annahmen logische Schlussfolgerungen abzuleiten«. Es wird anerkannt, dass mit dieser Methode rigorose logische Aussagen ermöglicht werden. Diese sind aber - so wird eingewendet - nicht ausreichend für wirtschaftspolitische Fragestellungen, da die mangelnde Logik und Vielfalt menschlicher Handlungsweisen und Institutionen nicht genügend berücksichtigt werden könnten. Immer mehr Ökonom|inn|en »opfern die Realitätsnähe ihrer Analyse dem Ziel formal-logischer Stringenz«. Hingegen sind »die Ergebnisse der wirtschaftspolitischen Analyse häufig kontrovers und - da sie empirischer Natur sind - nie mit letzter Gewissheit beweisbar«. Gefordert wird schließlich auf Grund dieser Betrachtungen die Aufrechterhaltung der bedrohten wirtschaftspolitischen Lehrstühle.

In einem etwas längeren Beitrag erfolgte dann die Stellungnahme der I88 Ökonom|inn|en im $\mathrm{HB}$, in der sie die geplante Umwidmung der wirtschaftspolitischen Lehrstühle befürworten. Ihr Aufruf will zwei Zielen dienen. Das "Zerrbild moderner ökonomischer Forschung«, das in der FAZ dargeboten wurde, zu korrigieren, und die »international nicht übliche Zementierung der nicht fruchtbaren Trennung an deutschen Fakultäten zwischen Wirtschaftstheorie und Wirtschaftspolitik« zu beseitigen. In der smodernen Volkswirtschaftslehre werden - so wird betont - theoretische und empirische Analyse, Theorie und Ökonometrie, gemeinsam eingesetzt, und sie behandelt sowohl die Funktion wie die Implikationen wirtschaftlicher Zustände und Entscheidungen. Es wird darauf hingewiesen, dass diese Kombination von Theorie und Empirie für den größten Teil der 
Arbeiten in internationalen 'Spitzenjournalen` ${ }^{3}$ typisch sei. Als weiteres Qualitätsmerkmal der Mainstream-Economics wird auf die Bedeutung von Mainstream-Ökonom|inn|en als wirtschaftspolitische Berater|innen US-amerikanischer Regierungen hingewiesen. Es wird zugegeben, dass die große Finanz- und Wirtschaftskrise neue Herausforderungen für die Volkswirtschaftslehre aufwirft, die eine aktive, sich entwickelnde Wissenschaft ist. Die gegenwärtige Forschung sucht nach einem tieferen Verständnis des Zusammenspiels von Finanz-, Banken-, und Realsektor und der Auswirkungen entsprechender wirtschaftspolitischer Instrumente. "Gute Theorie und gute Empirie werden hierbei eine wichtige Rolle spielen, alte Dogmen hingegen nicht. Die deutsche volkswirtschaftliche Forschung sollte von den wissenschaftspolitischen Entscheidungsträgern in den Stand versetzt werden, hier international maßgebende [gemeint sind 'Mainstream-konforme`, Anmerkung des Autors] Beiträge zu leisten."

Soweit die beiden öffentlichen Kundmachungen, die zu einer Kette weiterer Diskussionen führten, auf die aber hier nicht weiter eingegangen wird. Vielmehr geht es hier nur um einige Bemerkungen zu diesen beiden spektakulären Erklärungen in der Öffentlichkeit, zu diesem Duell FAZ contra HB. Zunächst soll betont werden, dass es sich bei dieser Auseinandersetzung nicht - wie im HB-Beitrag behauptet wird - um ein typisch deutsches Problem handelt, da nur an deutschen Universitäten eine »nicht fruchtbare Trennung « zwischen Wirtschaftstheorie und Wirtschaftspolitik bestehe und daher - so die HB-Forderung - eine Anpassung der volkswirtschaftlichen Fakultäten "an internationale Standards und Strukturen « notwendig sei. Diese Bemerkungen übersehen, dass zwar die Lehrstuhlbezeichnungen ' Wirtschaftstheorie und , Wirtschaftspolitikı eine deutsche Spezialität sein mögen (denen im Ausland eventuell Ausdrücke wie `Economic Theory` und `Applied Economics` gegenüberstehen), dass es aber im Wesentlichen um eine Auseinandersetzung zwischen verschiedenen Methoden geht; und in dieser Beziehung handelt es sich keineswegs um ein typisch deutsches Problem. Vielmehr passt diese Auseinandersetzung durchaus in eine seit Jahren intensiv verlaufende methodologische Diskussion zwischen ıorthodoxen Mainstream-Ökonom|inn|en und 'heterodoxen` Ökonom|inn|en und Wissenschaftstheoretiker|inne|n, an der allerdings deutsche Autor|inn|en nur schwach beteiligt sind. Eventuell kann man ein deutsches Element darin sehen, dass im FAZ-Text auch die systematische Analyse der normativen Grundlagen als essentieller Bestandteil der Wirtschaftsforschung erwähnt wird, der wohl von den Ordoliberalen in dieser Gruppe betont wurde, aber nicht entscheidend für das Gesamtkonzept ist. Die Grundgedanken der Debatte überschneiden sich im Großen und Ganzen mit den früheren, auf Menger/Schmoller zurückgehenden, und mit laufenden Konfrontationen. Dabei geht es um die Gegenüberstellung einer Präferenz für vorwiegend axiomatisch-deduktive Methoden mit Betonung möglichst rigoroser und möglichst quantitativer Resultate und `Gesetzmäßigkeiten` einerseits und andererseits einer mehr `offenen` Ausrichtung, welche die Dynamik und Komplexität der Wirtschaft und der ökonomischen Probleme als Begründung für einen größeren Einsatz von induktiven, deskriptiven und

3 Als Spitzenjournale werden genannt: American Economic Review, Econometrica, Journal of Political Economy, Quarterly Journal of Economics, Review of Economic Studies. 
interdisziplinären Elementen befürwortet, was zwar einen Verzicht auf rigoros-generelle Aussagen bedeutet, dafür aber die Berücksichtigung variierender und nicht quantifizierbarer Elemente erleichtert und damit eine größere Realitätsnähe ermöglicht.

Dieser traditionelle Hintergrund des sklassischen Methodenstreits kann aber aus der Perspektive zweier verschiedener Fragestellungen gesehen werden. Eine Frage ist die des ,Entweder/Oderı, das heißt, welche Methode die richtige ist und welche die falsche, die man dann aufgeben sollte. Ein weniger radikales Motiv für die Gegenüberstellung beruht auf einer pluralistischen Position, die angesichts der Komplexität des Wirtschaftslebens den Einsatz verschiedener Theorien und Methoden als notwendig erachtet, aber die Bevorzugung (`Mainstreamisierung`) einer Methode im Lehr- und Forschungsbetrieb kritisiert. In diesem zweiten Fall ist es also nicht nötig, die eine oder die andere Methode als ‘falsch oder sunwissenschaftlich ‘ abzuwerten, sondern es geht nur um die Frage ihrer relativen Bedeutung und Entwicklungsmöglichkeiten. Das Malheur ist nur, dass die Auseinandersetzungen auch im zweiten Fall häufig so heftig geführt werden, dass der Eindruck entsteht, es gehe um Fall I und das Ziel der totalen Vernichtung des Gegners. Dies war bereits in der Menger/ Schmoller-Auseinandersetzung der Fall. Wiewohl beide in ihren Schriften die Leistungen der 'gegnerischen`Schule im Prinzip anerkannten, ging es dann im Streit darum, ihre Rolle bis zur Bedeutungslosigkeit herunterzuspielen. Menger sprach von der historischen Schule als "Kärrner", der die Elemente für die Theorie herbeischafft, und Schmoller billigte der theoretischen Forschung ein »Eckchen« in der Wirtschaftswissenschaft zu. Übrig blieb Geschichte kontra Theorie.

Wenn man die sehr komprimierten FAZ/HB-Beiträge liest, so hat man das Gefühl, dass es um den Fall 2 geht, wiewohl in den nachfolgenden Diskusionen gelegentlich der ,Entweder/Oder-Standpunkt herausgelesen wird. Aber wer die beiden Texte vorurteilsfrei liest und keine engen Vorstellungen bezüglich , Wissenschaftlichkeit hegt (Feyerabend 1976), wird - je nach Einstellung - in beiden Stellungnahmen 'vernünftige` und sunvernünftige` Argumente finden. Es gibt keinen klaren Sieg für die eine oder andere Methode. ${ }^{4}$ Wohl aber geht es um die Frage der relativen Stellung des sdeduktiven` Mainstreams und der Heterodoxen (hier 'Wirtschaftspolitikı) im Lehr- und Forschungsbereich. Und es ist nicht uninteressant, die Debatte aus dieser Sicht kritisch zu betrachten.

Wenn man die beiden Stellungnahmen auf die Frage hin untersucht, welche Gruppe bezüglich der wünschenswerten Gewichtung 'theoretischer (Mainstream) und 'wirtschaftspolitischer` (heterodoxer) Richtungen im Lehr- und Forschungsbetrieb `recht hat‘, so könnte

4 An dieser Stelle mag es erlaubt sein, gänzlich unmethodisch einen alten jüdischen Witz zu erzählen. In einem galizischen Dorf kommt ein Ehepaar, das in einer Hütte am Rand des Dorfs wohnt, zum Rabbi, damit er einen Streit zwischen ihnen schlichte. Es geht darum, ob sie in diesem kalten Winter ihre einzige Ziege in die warme Stube nehmen oder frierend im Freien lassen sollen. Sagt der Mann: "Die Ziege muss draußen bleiben, sonst haben wir diesen fürchterlichen Gestank in der Stube». "Da hast du recht«, sagt der Rabbi. Nun sagt die Frau: „Nein, man muss die Ziege ins Haus nehmen. Man kann das arme Tier doch nicht so frieren lassen«. »Da hast du recht«, sagt der Rabbi. Darauf der Mann etwas erbost: »Erst sagst du, ich habe recht, dann sagt meine Frau das Gegenteil und du sagst, sie hat recht. Das ist doch ein Unsinn«. Sagt der Rabbi: »Da hast du recht«. 
auf den ersten Blick (wie es dann auch zum Teil geschieht) der HB-Gruppe ein eindeutiger Sieg zugesprochen werden. Denn diese hat ja mit ihren I88 Unterzeichner|inne|n eine klare Mehrheit gegenüber den 83 FAZ-ler|inne|n. Dies allein muss aber nicht viel bedeuten, erstens, weil , Wahrheit nicht durch Mehrheitsbeschlüsse entschieden werden kann, und zweitens, weil die insgesamt 27I Diskussionsteilnehmer|innen nicht notwendigerweise repräsentativ für die weit größere Zahl der Ökonom|inn|en ist, die in Lehre und Forschung tätig sind.

Aber da ist ein zweiter Faktor, der das obige Mehrheitsargument zu bestätigen scheint. Vergleicht man die beiden Listen der Unterzeichner|innen mit einer der RankingListen, die fallweise im HB erstellt werden (im Folgenden das im Jahr 2008 publizierte Ökonom|inn|en (VWL)-Ranking der 200 Top-Ökonom|inn|en im deutschen Sprachbereich auf Basis ihres Lebenswerks), so ergibt sich folgendes Bild. Erstens, dass von den insgesamt 27I Teilnehmer|inne|n an der Konfrontation nur $5 \mathrm{I}$ in der Ranking-Liste enthalten sind. Das heißt, dass nur ein Viertel dieser so genannten Top-Ökonom|inn|en beteiligt waren. So viel zur Repräsentativität. Aber besonders bedeutsam und auffällig ist, dass von diesen 5I Top-Ökonom|inn|en 47 die HB-Stellungnahme unterzeichneten, aber nur vier die FAZStellungnahme. In Anteilen ausgedrückt heißt das, dass 25 Prozent der HB-Gruppe, aber nur 4,8 Prozent der FAZ-Gruppe zu den Top-Ökonom|inn|en gehören`. Also nicht nur das Mengenargument, auch ein Qualitätsargument scheint den HB-Standpunkt zu bekräftigen, dass die bestehende und fortzusetzende Tendenz einer Gewichtsverschiebung zugunsten des vorherrschenden Mainstreams eine rrichtiger Schlussfolgerung aus der Diskussion ist. Diese Schlussfolgerung soll nun kritisch hinterfragt werden.

Der entscheidende Punkt bei dieser Frage ist der Umstand, dass die Konfrontation nicht zu Beginn einer Beratung zwischen zwei ungefähr gleich starken Gruppen erfolgte, sondern zu einem Zeitpunkt, zu dem eine Gruppe - der Mainstream - über viele Jahre hinweg ein Übergewicht gewonnen und ausgebaut hat. Das hat theoretische, methodologische und ideologische Ursachen, auf die hier nicht näher eingegangen werden muss. Es genügt, darauf hinzuweisen, dass in all diesen Jahren der Mainstream in Lehre und Forschung auf Kosten alternativer Richtungen forciert wurde. Das mag richtig oder 'falsch gewesen sein (darum geht es ja in der Auseinandersetzung), aber Tatsache ist, dass durch diese jahrelange Entwicklung und Indoktrination jetzt zwei ungleiche Gruppen einander gegenüberstehen, eine große Gruppe etablierter Mainstreamler|innen, die auf Grund ihrer erworbenen Wissensgrundlagen und ihren gegenwärtigen Karrieremöglichkeiten bewusst oder unbewusst die Mainstreamtendenzen verteidigen, und eine kleinere und zurückgedrängte Gruppe heterodoxer und alternativer Ansichten, die um die gleichen Möglichkeiten im Lehr- und Forschungsbereich kämpft. I88 HB-Unterzeichner|innen und nur 83 FAZUnterzeichner|innen sagt daher nichts über ırichtig oder 'falsch aus, sondern eher über die Tatsache jahrelanger `Züchtung und Bevorzugung von Mainstream-Ökonom|inn|en, die man nun im Lehr- und Forschungsbereich in größerer Zahl vorfindet.

5 Allerdings ist der Top-Ökonom Nr. I (Bruno Frey) Unterzeichner der FAZ-Erklärung. TopÖkonom Nr. 2 (Martin Hellwig) ist bei HB. 
Aber wie ist das mit der Qualitätsfrage? Auch hier zeigt sich bei näherer Untersuchung, dass die ungleichen Anteile der Top-Ökonom|inn|en in den beiden Gruppen mit dem bereits bestehenden Übergewicht des Mainstreams zu tun hat und keinen Beweis für die Berechtigung dieses Übergewichts darstellt. Denn das Ranking-Verfahren des Handelsblatts (gleiches gilt für viele andere Ranking-Methoden) impliziert bereits in seiner Methode eine Überlegenheit des Mainstreams. Das entscheidende Element in der Qualitätsevaluierung in diesem Ökonomen-Ranking ist die Anzahl der Veröffentlichungen in ökonomischen Fachzeitschriften. Das ist an sich schon nicht ganz befriedigend, weil die Veröffentlichungen sehr unterschiedliche Qualität besitzen und weil Forschungsbeiträge in Buchform (die gelegentlich mit einem geringeren Output von Zeitschriftartikeln verbunden sind) nicht die notwendige Beachtung finden. Aber darüber kann oder muss man hinwegsehen, ebenso wie über die Tatsache, dass nicht alle relevanten Zeitschriften erfasst werden können. Was aber bewusst eingeführt ist und kritisch hinterfragt werden muss, ist die verschiedene Bewertung der Journalbeiträge. Den Zeitschriften werden verschiedene Qualitätsnoten zugeteilt, und diese beeinflussen die Bewertung der verschiedenen Beiträge. Artikel in den `Top-Journalen erhalten einen Punkt, Artikel in allen anderen Zeitschriften erhalten Werte, die zwischen 0,67 und 0,2 Punkten liegen. Als Top-Journale gelten fünf Zeitschriften: American Economic Review, Econometrica, Journal of Political Economy, Review of Economic Studies und Quarterly Journal of Economics.

Nun braucht man sicher nicht zu bezweifeln, dass diese Top-Journale eine hohe Qualität besitzen. Aber es ist nicht schwer, auch anderen Zeitschriften eine hohe Qualität zuzubilligen, wie z.B. Economic Journal, Cambridge Journal of Economics, Kyklos, Weltwirtschaftliches Archiv, Journal of Post-Keynesian Economics usw. Was zusätzlich sehr wohl zutrifft, ist, dass die gewählten fünfTop-Journale die Glanzprodukte der Mainstream Economics sind. Sowohl Herausgeber|innen wie Referees sind überwiegend Mainstream, und Ökonom|inn|en anderer Richtungen finden nur selten Zugang zu diesen Zeitschriften, und/oder - was vielleicht ebenso entscheidend ist - versuchen es gar nicht mehr. Damit ergibt sich für das RankingVerfahren ein selbstverstärkender Prozess. Ein Mainstream, der sich aus theoretischen und ideologischen Gründen etabliert hat und bestimmte führende Zeitschriften dominiert, erklärt diese zu `Top-Journalen`. Ökonom|inn|en, die in diesen Journalen publizieren dürfen, werden bevorzugt zu Top-Ökonom|inn|en ernannt. Mit anderen Worten, es entsteht eine merkwürdige Doppelbeziehung: Top-Ökonom|inn|en sind Ökonom|inn|en, die in Top-Journalen publizieren, und Top-Journale sind Journale, in denen Top-Ökonom|inn|en publizieren. Aber was Top ist, ist bereits vorbestimmt.

Wie auch immer. Tatsache ist, dass die bedeutend stärkere Gewichtung der Beiträge in den Mainstream-Journalen automatisch dazu führt, dass deren Autor|inn|en im RankingVerfahren die besseren Plätze einnehmen können. Wenn man nicht von vornherein die Ansicht vertritt, dass der Mainstream die einzig richtige oder zumindest die einzig seligmachende Methode ist, dann besagt die weitaus stärkere Vertretung von 'führenden Ökonom|inn|en in der HB-Liste nichts über eine Überlegenheit dieser Gruppe, sondern ist weitgehend eine Folge des gewählten Rankingprozesses, also der vorweggenommenen Bevorzugung des Mainstreams. 
Also? Der alte fruchtlose Streit eines 'Entweder/Oder ist, wie schon Schumpeter in Zusammenhang mit der Menger/Schmoller-Auseinandersetzung sagte, »im Wesentlichen eine Geschichte vergeudeter Energie«(Schumpeter 1965: 994). Die Fragen bezüglich Pluralismus, Mainstream-Ansprüchen und dergleichen bleiben aktuell und Gegenstand fortlaufender Diskussionen. Die FAZ/HB-Konfrontation hat allerdings wenig dazu beigetragen.

\section{Literatur}

Feyerabend, P. (1976): Wider den Methodenzwang, Frankfurt/Main.

Marshall, A. (1920/1994): Principles of Economics, $8^{\text {th }}$ Edition, London: Macmillan.

Schumpeter, J.A. (1965): Geschichte der ökonomischen Analyse, Göttingen.

\section{Assessing economic research and the future of heterodox economics. Failures and alternatives of journals, departments, and scholars rankings Wolfram Elsner ${ }^{*}$ Fred Lee}

\section{Journal and department rankings and the ruling game of mainstream economics}

Evaluating economic research today is a most contested field. This applies, most notably, since individual careers of a whole generation of critical young economists are affected. And it applies in economics, perhaps more than in any other discipline, since it is the most important academic discipline for the ideological legitimization of capitalism and one of the few, perhaps the only, fundamentally divided and contested discipline. What the ruling forces of the economy, of professional politics, of science administration, and particularly of economic science have made out of the complex issues and processes of evaluating research quality is reducing them down to a simplistic, allegedly exact, objective, and obvious, but fundamentally mistaken procedure of a one-dimensional ranking of quantitative domination, a cumulative dictatorship of mass. And this is done in surprisingly unprofessional ways, subject to many obvious misconceptions and failures. For example, the

* Professor of Economics, University of Bremen, Faculty of Business Studies and Economics, iino Institute for Institutional and Innovation Economics.

** Professor of Economics, Department of Economics, University of Missouri at Kansas City.

(C) INTERVENTION 7 (I), 20IO, 3I-4I 